\title{
Valuation of Quanto Floating Range Notes under the Cross-Currency LIBOR Market Model
}

\author{
Chi-Hsun Chou ${ }^{1}$, Tsung-Yu Hsieh ${ }^{2} \&$ Son-Nan Chen ${ }^{3}$ \\ ${ }^{1}$ Department of Management, Fo Guang University, Yilan County, Taiwan (R.O.C.) \\ ${ }^{2}$ Department of Banking and Finance, Tamkang University, New Taipei City, Taiwan (R.O.C.) \\ ${ }^{3}$ Shanghai Advanced Institute of Finance, Shanghai Jiao Tong University, Shanghai, China \\ Correspondence: Chi-Hsun Chou, Department of Management, Fo Guang University, No.160, Linwei Rd., \\ Jiaosi , Yilan County 26247, Taiwan (R.O.C.). Tel: 886-3-987-1000 ext 23818. E-mail: cschou@ mail.fgu.edu.tw
}

Received: September 13, 2015

Accepted: October 30, $2015 \quad$ Online Published: November 25, 2015

doi:10.5539/ijef.v7n12p70

URL: http://dx.doi.org/10.5539/ijef.v7n12p70

\begin{abstract}
In this paper, we propose analytical valuation formulae for three types of quanto floating range notes based on the cross-currency LIBOR market model. The dynamics of forward LIBOR rates is a multifactor model that incorporates both the domestic and foreign interest rate process and the exchange rate process in a cross-currency environment. The derived formulae are analytically tractable and easy to implement in practice. The model parameters can be extracted directly from market quantities. We show that the empirical results are more accurate and robust than the results of Monte Carlo simulation.
\end{abstract}

Keywords: cross-currency LIBOR market model, delayed digital range options, delayed asset-or-nothing range options, quanto floating range notes

\section{Introduction}

In this paper, we attempt to derive analytical valuation formulae for quanto floating range notes (QFRNs) based on the cross-currency LIBOR market model (CLMM), which was introduced by Wu et al. (2007), who extended the Brace et al. (1997) approach. The CLMM has a cross-currency setting and incorporates both the domestic and foreign interest rate process and the exchange rate process.

Floating range notes (FRNs) are structured notes with coupon payments dependent on the level of interest rates (most commonly the LIBOR rates, e.g., 3-month LIBOR rates). Similar to standard floating rate notes, FRNs offer investors floating coupon payments periodically at the end of each period (paid in arrears) based on the level of one LIBOR rate specified at the start of each period (reset in advance). However, coupon payments are also calculated in proportion to the number of days that another reference LIBOR rate lies within a determined range (corridor) during each compounding period. To avoid ambiguity, the former LIBOR rate reset in advance is called "coupon payment rate" (CP rate), while the other rate, which is associated with a corridor, is called "reference interest rate" (RI rate). These products provide investors with the opportunity to enhance yield in a low-interest-rate environment based on the belief that the CP rate will stay within the corridor. It usually offers rates with spreads higher than standard floating rate notes. In return, the investors run the risk of no coupon paid for the time the corridor remains. Notably distinct from FRNs, QFRNs are cross-currency structured products in which $\mathrm{CP}$ rates and RI rates are based on the LIBOR rates of two countries rather than on domestic currency rates only. In QFRNs, the notional principal is denominated in the domestic currency to avoid the direct exposure of currency risk from the domestic investors' perspective. Three different types of QFRNs are examined in this paper. The coupon payments of first-type QFRNs are calculated in terms of foreign CP rates and foreign RI rates. In contrast, the coupon payments of the second- or third-type QFRNs are associated with foreign (or domestic) $\mathrm{CP}$ rates and domestic (or foreign) RI rates. For example, the second-type QFRNs entitle the investor to receive coupon payments at the end of each period. The payments depend on the foreign CP rates at the beginning of each period and are proportional to the numbers of day on which the domestic RI rates lie inside a pre-specified corridor during each period.

Some researchers have documented the valuation of FRNs and QFRNs. Turnball (1995) derived the valuation formulae for FRNs in the one-factor Gaussian Heath-Jarrow- Morton framework (hereafter, Gaussian HJM 
framework). Navatte et al. (1999) followed the one-factor Gaussian HJM framework to demonstrate the solution in a more intuitive way. They decomposed a FRN into a portfolio of a set of options called double delayed digital options. Nunes (2004) adopted the multifactor Gaussian HJM framework to provide an FRN pricing model, which was also regarded as a portfolio of delayed digital options. Eberlein et al. (2006) derived an analytical valuation formula for FRNs that generalized the multifactor Gaussian HJM model with a multivariate Lévy process. Wu et al. (2008) provided a general pricing formula for FRNs in the framework of the LIBOR market model. Liao et al. (2009) analyzed the pricing and hedging problems for QFRNs under the HJM framework with a Lévy process for instantaneous domestic and foreign forward interest rates.

Compared to the aforementioned studies, the valuation formulae of QFRNs derived in this article have several advantages.

First, previous pricing formulae cannot easily transform traded quantities into model parameters; hence, they are not easy to implement in practice. In addition, illustrated applications are relatively rare in previous studies. In Section 4, we will use market data from the U.S. and the U.K. to calibrate the model parameters and demonstrate accurate numerical results for practitioners.

Second, the pricing formulae are shown to be analytically tractable, thereby leading to pricing efficiency and precision for practical implementation. Because the payoffs of QFRNs have a path-dependency relation, they are difficult to implement in practice. In contrast, the model developed in this paper is advantageous in that it does not involve complicated settings inherent in other abstract interest rate models, time-consuming Monte Carlo simulations, and other numerical analyses that prevent the products' issuers and dealers from using them for application.

Third, forward LIBOR rates have a lognormal volatility structure, which prevents them from becoming negative with positive probability.

Fourth, the pricing formulae of previously presented QFRNs are more of a general case. In fact, the pricing model of FRNs developed in Wu et al. (2008) is referenced in our paper.

This paper is organized as follows. Section 2 briefly reviews the arbitrage-free CLMM as well as the dynamic processes of the foreign and domestic LIBOR rates and exchange rates. In Section 3, the valuation formulae of QFRNs are developed and decomposed into a series of options. Section 4 provides a calibration procedure for practical operation and numerical examples to demonstrate the working of the model. The last section presents the concluding remarks.

\section{The Model}

In this section, we briefly introduce the arbitrage-free CLMM and list the notations used in our model. Assume that trading occurs continuously over an interval $[0, \mathbf{T}], 0<\mathbf{T}<\infty$. The uncertainty is described by the filtered probability space $\left(\Omega, \mathbf{F}, Q,\{F\}_{t \in[0, T]}\right)$, where the filtration is generated by independent standard Brownian motions $W(t)=\left(W_{1}(t), W_{2}(t), \ldots, W_{m}(t)\right)$. The element $Q$ represents the domestic spot martingale probability measure. There are some domestic and foreign assets in this economy. Hereafter, the subscript $k \in\{d, f\}$ represents the $k$ th country's asset with $d$ for domestic and $f$ for foreign.

$L_{k}(t, T, \delta)=$ the $k$ th country's forward interest rate contracted at time $t$ (or today) and effective at time $T$ for a simple compounded period $[T, T+\delta]$ with $0 \leq t \leq T \leq \mathbf{T}$.

$P_{k}(t, T)=$ the time $t$ price of the $k$ th country's zero-coupon bond paying one currency unit at maturity $T$.

$r_{k}(t)=$ the $k$ th country's risk-free short rate at time $t$.

$\beta_{k}(t)=\exp \left[\int_{0}^{t} r_{k}(s) d(s)\right]$, the $k$ th country's money market account at time $t$ with initial value $\beta_{k}(0)=1$.

$X(t)=$ the spot exchange rate at $t \in[0, \mathbf{T}]$ for one unit of the foreign currency expressed in terms of the units 
of the domestic currency.

The forward LIBOR rate $L_{k}(t, T, \delta)$ is related to the zero-coupon bond $P_{k}(t, T)$. By fixing some accrual periods, the forward LIBOR rate can be defined as follows:

$$
1+\delta L_{k}(t, T, \delta)=\frac{P_{k}(t, T)}{P_{k}(t, T+\delta)}
$$

where $\delta$ is the length of a compounding period $[T, T+\delta]$.

Under the CLMM, the dynamics of assets are expressed under the risk-neutral probability measure $Q$, which is induced by the domestic money market account $\beta_{d}(t)$. The domestic and foreign forward LIBOR rates $L_{k}(t, T, \delta)$ and the exchange rates $X(t)$ are denominated in terms of the units of $\beta_{d}(t)$ in the model. The drift and volatility terms of the dynamics of assets are determined by the arbitrage-free relationship. The following assumption introduced by Wu et al. (2007) briefly describes the pricing framework.

Assumption 1. The asset dynamics under the measure $Q$

For any time $T \in[0, \mathbf{T}]$, the dynamics of the forward LIBOR rates $L_{k}(t, T, \delta)$ and the exchange rate $X(t)$ under the domestic martingale measure $Q$ are given as follows:

$$
\begin{gathered}
\frac{d L_{d}(t, T, \delta)}{L_{d}(t, T, \delta)}=\gamma_{d}(t, T) \cdot \sigma_{d}(t, T+\delta) d(t)+\gamma_{d}(t, T) \cdot d W(t) \\
\frac{d L_{f}(t, T, \delta)}{L_{f}(t, T, \delta)}=\gamma_{f}(t, T) \cdot\left(\sigma_{f}(t, T+\delta)-\sigma_{X}(t)\right) d(t)+\gamma_{f}(t, T) \cdot d W(t) \\
\frac{d X(t)}{X(t)}=\left(r_{d}(t)-r_{f}(t)\right) d(t)+\sigma_{X}(t) \cdot d W(t)
\end{gathered}
$$

where $t \in[0, T] . \quad \gamma_{k}(t, T)$ and $\sigma_{k}(t, T)$ denote the volatility processes of the $k$ th country's forward LIBOR rate $L_{k}(t, T, \delta)$ and the zero-coupon bond $P_{k}(t, T)$, respectively. $\sigma_{X}(t)$ stands for the volatility process of the spot exchange rate $X(t)$.

To derive the pricing formulae of QFRNs, there are two main points to be determined in Assumption 1. First, the zero-coupon bond volatility process $\left\{\sigma_{k}(t, T)\right\}$ is stochastic rather than deterministic, which makes dynamics (1) and (2) difficult to solve for the forward rate $L_{k}(t, T, \delta)$. Instead, according to the recurrence relation between $\sigma_{k}(t, T+\delta)$ and $\gamma_{k}(t, T)$, the element $\sigma_{k}(t, T)$ can be approximated by $\bar{\sigma}_{k}^{\tau}(t, T)$ with a fixed initial time $\tau$, which is defined by

$$
\bar{\sigma}_{k}^{\tau}(t, T)=\sum_{i=1}^{\left[\delta^{-1}(T-t)\right]} \frac{\delta L_{k}(\tau, T-i \delta, \delta)}{1+\delta L_{k}(\tau, T-i \delta, \delta)} \gamma_{k}(t, T-i \delta)
$$

where $t \in[\tau, T-\delta]$, and $T-\delta>0$. It means that the calendar time of the process $\left\{L_{k}(s, T-i \delta, \delta)\right\}_{s \in[\tau, T-i \delta]}$ is frozen at its initial time $\tau$. By substituting (4) into the drift and volatility terms in (1) and (2), the resulting equations become solvable, and the distribution of the forward LIBOR rate is approximated as lognormally distributed.

Second, the pricing process of QFRNs is relatively easier to conduct under the domestic forward measure $Q^{T}$ than under the risk neutral measure $Q$. The forward measure $Q^{T}$ is induced by the domestic zero-coupon bond $P_{d}(t, T)$ as the numéraire. By using the changing-numéraire mechanism, the drift and volatility terms of the assets dynamics under the forward measure $Q^{T}$ can be specified. The result is shown in Assumption $2^{1}$. 
Assumption 2. The approximated asset dynamics under the measure $Q^{T}$

For any time $S, T \in[0, \mathbf{T}], S \leq T$, the dynamics of the forward LIBOR rates $L_{k}(t, S, \delta)$ and the exchange rate $X(t)$ under the forward martingale measure $Q^{T}$ are given as follows:

$$
\begin{gathered}
\frac{d L_{d}(t, S, \delta)}{L_{d}(t, S, \delta)}=\gamma_{d}(t, T) \cdot\left(\bar{\sigma}_{d}^{\tau}(t, S+\delta)-\bar{\sigma}_{d}^{\tau}(t, T)\right) d(t)+\gamma_{d}(t, S) \cdot d W^{T}(t) \\
\frac{d L_{f}(t, S, \delta)}{L_{f}(t, S, \delta)}=\gamma_{f}(t, T) \cdot\left(\bar{\sigma}_{f}^{\tau}(t, S+\delta)-\bar{\sigma}_{d}^{\tau}(t, T)-\sigma_{X}(t)\right) d(t)+\gamma_{f}(t, S) \cdot d W^{T}(t) \\
\frac{d X(t)}{X(t)}=\left(r_{d}(t)-r_{f}(t)-\sigma_{X}(t) \cdot \bar{\sigma}_{d}^{\tau}(t, T)\right) d(t)+\sigma_{X}(t) \cdot d W^{T}(t)
\end{gathered}
$$

where $t \in[0, T]$ and $\bar{\sigma}_{k}^{\tau}(t, T)$ is defined in (4).

In Assumption 2, the drift term of the forward LIBOR rate $L_{k}(t, S, \delta)$ is then specified by the approximated zero-coupon bond volatility $\bar{\sigma}_{k}^{\tau}(t, T)$ in (4). The volatility term remain unchanged under the forward measure $Q^{T}$. By avoiding the stochastic characteristic, the distribution of the forward LIBOR rate $L_{k}(t, S, \delta)$ is lognormally distributed.

In addition, the reset time $S$ of the forward LIBOR rate $L_{k}(t, S, \delta)$ in Assumption 2 is set before the forward measure time $T$, that is, $S \leq T$. This setting is used because each RI rate is reset prior to each coupon payment date during each compounding period. Hence, it is convenient to treat the pricing problem with the dynamic process of $L_{k}(t, S, \delta)$ under the forward measure $Q^{T}$.

In practice, the volatility of the forward LIBOR rates $\gamma_{k}(t, T)$ and the approximated bond volatility $\bar{\sigma}_{k}^{\tau}(t, T)$ are easy to be calibrated under the CLMM. Specifically, they can be extracted via the model from quoted prices in financial markets, such as caps and floors. Once the parameters are calibrated, the pricing formulae under the CLMM are analytically tractable for practical application.

After the above model presentation, the pricing formulae of QFRNs are shown in Section 3.

\section{Valuation of Quanto Floating Range Notes}

Three different types of QFRNs are considered in this section. Based on the CLMM, we next derive the analytical valuation models of QFRNs. Each coupon is based on the level of CP rates and proportional to the number of days on which RI rates lie inside a corridor in each compounding period. The definitions and notations are listed as follows:

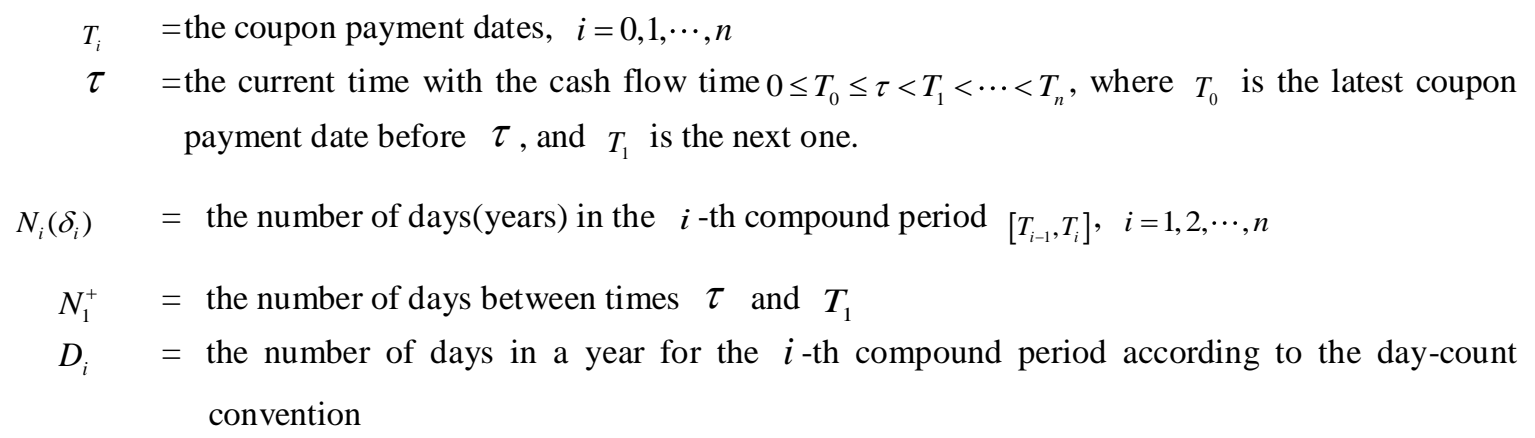


$T_{i j} \quad=$ the date corresponding to $j$ days after time $T_{i}$ where $i=0,1, \cdots, n-1, j=0,1, \cdots, N_{i+1}$ and $T_{i, N_{i+1}}=T_{i+1}$

$\delta_{i j} \quad=\quad$ the compounding length of the RI rate observed at time $T_{i j}$

$C_{i}(t)=$ the time $t$ value of the $i$-th coupon payment paid at time $T_{i}$

$H_{i j}=\left[H_{i j}^{L}, H_{i j}^{U}\right]$, the corridor level with the lower bound $H_{i j}^{L}$ and the upper bound $H_{i j}^{U}$

$\Delta_{i j} \quad=$ the spread employed for date $T_{i j}$

\section{Definition 1. QFRNs}

The coupon payments associate three types of QFRNs with CP rates $L_{s}\left(T_{i}, T_{i}, \delta_{i}\right)$ and RI rates $L_{k}\left(T_{i j}, T_{i j}, \delta_{i j}\right)$ are paid in domestic currency and defined as follows, where $s, k \in\{d, f\}$. The first coupon payment at date $T_{1}$ is given by

$$
C\left(T_{1}, T_{1}, H_{0 j}, s, k\right)=\frac{V\left(T_{0}, \tau\right)+\sum_{j=1}^{N_{1}^{+}}\left[L_{s}\left(T_{0}, T_{0}, \delta_{0}\right)+\Delta_{0 j}\right] \times I\left(T_{0 j}\right)}{D_{0}}
$$

where $V\left(T_{0}, \tau\right)$ is the realized-payoff amount between times $T_{0}$ and $\tau$. The $(i+1)$ th coupon payment at date $T_{i+1}$ for $i=1,2, \cdots, n-1$ is given by:

$$
C\left(T_{i+1}, T_{i+1}, H_{i j}, s, k\right)=\frac{\sum_{j=1}^{N_{i}}\left[L_{s}\left(T_{i}, T_{i}, \delta_{i}\right)+\Delta_{i j}\right] \times I\left(T_{i j}\right)}{D_{i}}
$$

$I\left(T_{i j}\right)$ is an indicator function for $i=0,1, \cdots, n-1, \quad j=0,1, \cdots, N_{i}$

$$
I\left(T_{i j}\right)=\left\{\begin{array}{cc}
1, & H_{i j}^{L} \leq L_{k}\left(T_{i j}, T_{i j}, \delta_{i j}\right) \leq H_{i j}^{U} \\
0, & \text { otherwise }
\end{array}\right.
$$

The coupon payments in (8) and (9) represent three types of QFRNs depending on the following cases:

(1) The first-type of QFRNs, where $s=f$ and $k=f$

(2) The second-type of QFRNs, where $s=f$ and $k=d$

(3) The third-type of QFRNs, where $s=d$ and $k=f$

The principal is assumed to be one dollar, paid at the last payment date $T_{n}$.

For the domestic investors, the domestic currency-denominated coupons of the first-type QFRNs are linked to the foreign LIBOR rate. It provides an opportunity to profit when the investors expect a relative change in the term structures of interest rates in different countries. For example, if a domestic investor expects that the shape of the foreign yield curve might sharpen relative to the domestic one, the investor could change the investment from an outstanding FRN into one of the first-type QFRNs. Thus, he would receive higher coupon payments in domestic principal. Similarly, a corporate issuer might want to reduce interest payments by linking to a lower foreign LIBOR rate, while a fund manager might enhance coupon yield with a higher foreign LIBOR rate. For investment banks, this product can be designed for customers to accommodate special cases.

The second- and third-type QFRNs are also denominated in domestic principal, thus avoiding a direct exposure of currency risk. A domestic investor might benefit from using these two types of products. First, he could receive a higher coupon payment rate from the differential of interest rates between domestic and foreign countries. Second, the coupon payment is proportional to the number of days in the compounding period of which the reference rate lies inside a corridor. A domestic investor would receive higher coupon payments when 
the dynamic of RI rates is stable. This means that a lower volatility level of RI rates is beneficial to the domestic investor. For corporate issuers and fund managers, the products could be designed to suit different purposes, such as to enhance coupon yields or reduce interest payments.

Previous research demonstrates that a FRN is a combination of some interest rate digital options and asset-or-nothing options. This also is true for QFRNs. We will introduce delayed range digital options and delayed asset-or-nothing range options. The QFRN pricing formulae will be derived according to the definition of these two options, given in Section 3.1 and Section 3.2.

\subsection{Delayed Digital Range Options}

At maturity date $T^{+}$, a delayed digital range option (DDRO) pays one domestic dollar if the RI rate reset at the previous observed time $T, T \leq T^{+}$lies between the pre-specified ranges. The time $T^{+}$value of a DDRO is given by

$$
\operatorname{DDRO}\left(T^{+}, T^{+}, H, k\right)=\left\{\begin{array}{cc}
1, & H^{L} \leq L_{k}(T, T, \delta) \leq H^{U} \\
0, & \text { otherwise }
\end{array}\right.
$$

where the RI rate $L_{k}(T, T, \delta)$ is the forward LIBOR rate reset previously at time $T$ with the compounding period $\delta \cdot H=\left[H^{L}, H^{U}\right]$ as the level of a corridor.

Proposition 1. The Pricing Formula of a DDRO

Under the CLMM, the no-arbitrage value of a DDRO, referred to the RI rate $L_{k}(T, T, \delta)$ with maturity $T^{+}$ and corridor $H$ at current time $\tau$, is given by:

$$
\operatorname{DDRO}\left(\tau, T^{+}, H, k\right)=P_{d}\left(\tau, T^{+}\right)\left\{N\left[x\left(H^{L}, k\right)\right]-N\left[x\left(H^{U}, k\right)\right]\right\}
$$

where

$$
x(*, k)=\frac{\ln \left[\frac{L_{k}(\tau, T, \delta)}{*}\right]+\rho_{k}(\tau, T, \delta)-\frac{1}{2} v_{k}(\tau, T)}{v_{k}(\tau, T)}
$$

(1) when the RI rate is the domestic LIBOR rate, $k=d$

$$
\begin{gathered}
\rho_{d}(\tau, T, \delta)=\int_{\tau}^{T} \gamma_{d}(t, T) \cdot\left[\bar{\sigma}_{d}^{\tau}(t, T+\delta)-\bar{\sigma}_{d}^{\tau}\left(t, T^{+}\right)\right] d(t) \\
v_{d}(\tau, T)=\int_{\tau}^{T}\left\|\gamma_{d}(t, T)\right\|^{2} d(t)
\end{gathered}
$$

(2) when the RI rate is the foreign LIBOR rate, $k=f$

$$
\begin{gathered}
\rho_{d}(\tau, T, \delta)=\int_{\tau}^{T} \gamma_{f}(t, T) \cdot\left[\bar{\sigma}_{f}^{\tau}(t, T+\delta)-\bar{\sigma}_{d}^{\tau}\left(t, T^{+}\right)-\sigma_{X}(t)\right] d(t) \\
v_{f}(\tau, T)=\int_{\tau}^{T}\left\|\gamma_{f}(t, T)\right\|^{2} d(t)
\end{gathered}
$$

Proof. See Appendix A

\subsection{Delayed Asset-or-Nothing Range Options}

At maturity date $T^{+}$, a delayed asset-or-nothing range option (DARO) pays the level of the CP rate reset at the previous time $T^{-}$in domestic currency, if the RI rate reset at time $T, T^{-} \leq T \leq T^{+}$lies within a pre-specified range. The time $T^{+}$value of a DARO is given by

$$
\operatorname{DARO}\left(T^{+}, T^{+}, H, s, k\right)=\left\{\begin{array}{cc}
L_{s}\left(T^{-}, T^{-}, \eta\right), & H^{L} \leq L_{k}(T, T, \delta) \leq H^{U} \\
0, & \text { otherwise }
\end{array}\right.
$$


where the CP rate $L_{s}\left(T^{-}, T^{-}, \eta\right)$, with a compounding period $\eta$ and the RI rate $L_{k}(T, T, \delta)$ with a compounding length $\delta$, are the forward LIBOR rates reset previously at time $T^{-}$and $T$, respectively. The subscript $S$ is explained as $s, k \in\{d, f\} \cdot H=\left[H^{L}, H^{U}\right]$ is the level of a corridor.

Proposition 2. The Pricing Formula of a DARO

Under the CLMM, the no-arbitrage value of a DARO referred to the CP rate $L_{s}\left(T^{-}, T^{-}, \eta\right)$ and RI rate $L_{k}(T, T, \delta)$ with maturity $T^{+}$and corridor $H$ at current time $\tau$ is given by

$$
\operatorname{DARO}\left(\tau, T^{+}, H, s, k\right)=P_{d}\left(\tau, T^{+}\right) L_{s}\left(\tau, T^{-}, \eta\right) e^{\rho_{s}\left(\tau, T^{-}, \eta\right)}\left\{N\left[y\left(H^{L}, k\right)\right]-N\left[y\left(H^{U}, k\right)\right]\right\}
$$

where

$$
y(*, k)=\frac{\ln \left[\frac{L_{k}(\tau, T, \delta)}{*}\right]+\varphi_{k}(\tau, T, \delta)-\frac{1}{2} v_{k}(\tau, T)}{v_{k}(\tau, T)}
$$

and where $\rho_{s}\left(\tau, T^{-}, \eta\right)$ and $v_{k}(\tau, T)$ are defined in (12)-(15).

(1) when the RI rate is the domestic LIBOR rate, $k=d$

$$
\varphi_{d}(\tau, T, \delta)=\int_{\tau}^{T} \gamma_{d}(t, T) \cdot\left[\bar{\sigma}_{d}^{\tau}(t, T+\delta)-\bar{\sigma}_{d}^{\tau}\left(t, T^{+}\right)+\gamma_{d}\left(t, T^{-}\right)\right] d(t)
$$

(2) when the RI rate is the foreign LIBOR rate, $k=f$

$$
\varphi_{f}(\tau, T, \delta)=\int_{\tau}^{T} \gamma_{f}(t, T) \cdot\left[\bar{\sigma}_{f}^{\tau}(t, T+\delta)-\bar{\sigma}_{d}^{\tau}\left(t, T^{+}\right)-\sigma_{X}(t)+\gamma_{f}\left(t, T^{-}\right)\right] d(t)
$$

Proof. See Appendix B.

\subsection{Quanto Floating Range Notes}

According to Definition 1, all the three types of QFRNs can be structured by the DDROs and DAROs. Hence, the pricing formulae are the summation of the equations derived in the above section. The valuation formulae of the QFRNs are shown below.

Proposition 3. The Pricing Formulae of QFRNs

By Definition 1, the coupon payments of these three types of QFRNs are referred to CP rates $L_{s}\left(T_{i}, T_{i}, \delta_{i}\right)$ and RI rates $L_{k}\left(T_{i j}, T_{i j}, \delta_{i j}\right)$. Under the CLMM, the no-arbitrage value of the first coupon payment with payment date $T_{1}$ and corridor $H_{0 j}$ at current time $\tau$ is given by

$$
C\left(\tau, T_{1}, H_{0 j}, s, k\right)=\frac{P_{d}\left(\tau, T_{1}\right) V\left(T_{0}, \tau\right)+\sum_{j=1}^{N_{1}^{+}}\left[L_{s}\left(T_{0}, T_{0}, \delta_{0}\right)+\Delta_{0 j}\right] D D R O\left(\tau, T_{1}, H_{o j}, k\right)}{D_{0}}
$$

where $V\left(T_{0}, \tau\right)$ is the realized-payoff amount between times $T_{0}$ and $\tau$. And the no-arbitrage value of the $(i+1)$ th coupon payment with payment date $T_{i+1}$ and corridor $H_{i j}$ at current time $\tau$ is given by

$$
C\left(\tau, T_{i+1}, H_{i j}, s, k\right)=\frac{\sum_{j=1}^{N_{i}}\left[\operatorname{DARO}\left(\tau, T_{i+1}, H_{i j}, s, k\right)+\Delta_{i j} \operatorname{DDRO}\left(\tau, T_{i+1}, H_{i j}, k\right)\right]}{D_{i}}
$$

where $i=1,2, \cdots, n-1$. The no-arbitrage value of a QFRN is given by

$$
Q F R N=\sum_{i=0}^{n-1} C\left(\tau, T_{i+1}, H_{i j}, s, k\right)+P_{d}\left(\tau, T_{n}\right)
$$


The second term on the right hand side of equation (22) is the price of the domestic zero-coupon bond paying one dollar at maturity $T_{n}$ and

(1)The first-type of QFRNs is in the case with $s=f$ and $k=f$

(2)The second-type of QFRNs is in the case with $s=f$ and $k=d$

(3)The third-type of QFRNs is in the case with $s=d$ and $k=f$

Proof. The result is derived directly using Definition 1, which says that a QFRN consists of DDROs and DAROs.

In equations (20) and (21), the coupon payment at each compounding period is developed from Proposition 1 and 2 due to the linear relationship. The fair value of QFRNs at current time therefore includes all coupon payments and the zero-coupon bond. Thus, the derived pricing formulae contain some advantageous characteristics.

The pricing model in Proposition 3 is constructed under the CLMM framework. As mentioned in Section 2, it is a good fit to the observed market data and circumvents the difficulty of transforming traded quantities into model parameters. In QFRNs, the payoffs directly depend on the level of the forward LIBOR rates. It can be easily drawn from the market and then applied to Proposition 3, thereby avoiding converting abstract interest rates in early short-rate based models and forward-rate based models. Moreover, the forward LIBOR rates have a lognormal volatility structure, which prevents interest rates from becoming negative with positive probability. In addition, the model parameters in the derived pricing formulae, such as the volatility of the forward LIBOR rates $\gamma_{k}(t, T)$ and the approximated bond volatility $\bar{\sigma}_{k}^{\tau}(t, T)$, can be calibrated conveniently from quoted caps and floor prices in financial markets.

The pricing formulae are analytically tractable and easily provide expedited and accurate solutions. As mentioned above, coupon payments in OFRNs are proportional to the number of days in which RI rates stay in a corridor during each compounding period. This path-dependency feature makes it difficult to implement in practice using time-consuming numerical analysis, thereby preventing the products' issuers and dealers from using it for applications. In contrast, the model developed in Proposition 3 is efficient and robust. The empirical analysis in the next section will demonstrate that the results are sufficiently accurate for practitioners.

In addition, the forward LIBOR rates from different countries are incorporated simultaneously in the model. The pricing formulae of QFRNs provide a general case. In fact, the pricing model of FRNs developed in Wu et al. [12] is nested in our paper with $s=d$ and $k=d$.

\section{Calibration and Numerical Analysis}

This section provides practitioners with a calibration procedure and numerical examples.

\subsection{Calibration Procedure}

The calibration of an interest rate model is one of the significant parts of its implementation. Given observable forward LIBOR rates and market-quoted volatilities of caplets, the pricing parameters in the pricing framework are virtually obtainable and immediate. The calibration methodology presented by Rebonato (1999) is employed in our paper. We perform a simultaneous calibration of the CLMM to the instantaneous total volatilities and correlation surface of the underlying forward LIBOR rates and the exchange rate. To illustrate the procedure, we assume there are $n$ assets in a cross-currency economy. The assets include $(n-1) / 2$ forward LIBOR rates of the $k$ th country and the exchange rate. The number of random shocks to the term structure of the forward rates, denoted by $m$, is determined based on a compromise between simplicity and accuracy in practical application. Here, we employ three stylized factors $(m=3)$, which are level, slope, and curvature of the term structure of interest rates.

The steps of calibration follow. First, the instantaneous volatility of the forward LIBOR rate $\gamma_{k}(t, T)$ is chosen such that the caplet price is correctly recovered. In other words, the market-quoted volatility for $T$ years from current time $\tau$ caplet, denoted by $\sigma_{i m p}(T)$, is given by:

$$
\int_{\tau}^{T+\delta}\left\|\gamma_{k}(t, T+\delta)\right\|^{2} d(t)=\delta_{i m p}^{2}(T) T
$$


Next, we assume that the current market term structure is recovered by a piecewise-constant instantaneous total volatility depending on only the duration of the underlying rates. The time-dependent volatility of each period for each rate can be determined by the stripping method presented in Rebonato (1999). To describe the volatility structure engaged in the CLMM, the element $S(i)$ is defined by

$$
\sum_{k=1}^{i} S(i)=\sigma_{i m p}^{2}(T) T
$$

where the subscript $i$ labels the $i$ th forward LIBOR rate, thus converting the instantaneous volatility into a time-dependent volatility structure. The element $S(i)$ is solved by the recurrence relation shown below

$$
S \equiv\left(\begin{array}{ccccc}
S_{1} & & & & \\
S_{2} & S_{1} & & & \\
S_{3} & S_{2} & S_{1} & & \\
\vdots & \vdots & \vdots & \ddots & \\
S_{n} & S_{n-1} & S_{n-2} & \cdots & S_{1}
\end{array}\right)
$$

Moreover, the instantaneous volatility of the exchange rate can be extracted from the on-the-run currency options prices in practice or calculated directly from historical data of the underlying exchange rate in the market. For simplicity, we assume that the term structure of volatility is flat.

Second, the correlation structure of the underlying rates in Assumption 2 is given by

$$
d W^{T}(t) d W^{T}(t)=\sum d(t)
$$

where $\Sigma$ is a correlation matrix, which represents the market correlation of the underlying rates. $\Sigma$ is an $n$ -rank, positive-definite, and symmetric matrix and can be written as follows:

$$
\sum=\mathbf{A A}^{\prime}=\mathbf{P A P}
$$

where $\mathrm{P}$ is a real orthogonal matrix and $\Lambda$ is a diagonal matrix. A is defined by

$\mathbf{A} \equiv \mathbf{P} \Lambda^{1 / 2}$. Next, the full-rank correlation matrix $\Sigma$ is approximated by selecting a suitable correlation matrix $\sum^{\mathbf{B}}$ that can be decomposed by an $m$-rank matrix B.

$$
\mathbf{B} d Z(t)(\mathbf{B} d Z(t))^{\prime}=\mathbf{B B}^{\prime} d(t)=\sum^{\mathbf{B}} d(t)
$$

The appropriate choice of the matrix B can be proposed by Rebonato (1999) and shown as:

$$
b_{i, k}=\left\{\begin{array}{cc}
\cos \theta_{i, k} \prod_{j=1}^{k-1} \sin \theta_{i, j} & k=1,2, \ldots, m-1 \\
\prod_{j=1}^{k-1} \sin \theta_{i, j} & k=m
\end{array}\right.
$$

Then, a suitable matrix $\hat{\mathbf{B}}$ is obtained by finding the solution $\hat{\theta}$ of the optimization problem.

$$
\min _{\theta} \sum_{i, j=1}^{n}\left|\Gamma_{i, j}^{\mathbf{B}}-\Gamma_{i, j}\right|^{2}
$$

Hence, the approximated correlation matrix $\sum^{\mathbf{B}}=\hat{\mathbf{B}} \hat{\mathbf{B}}^{\prime}$ mimics the correlation of the market $\Sigma$.

\subsection{Numerical Analysis}

Next, we provide numerical examples to illustrate the application of Proposition 3. Compared to previous studies, the parameters in our model are calibrated by observable quantities from market data to examine model efficiency and accuracy.

The accuracy of the pricing formulae will be compared to that of Monte Carlo simulation. In addition, we consider the QFRNs of short and long maturities with different levels of corridors to examine the robustness of the pricing models.

The market data - associated with the forward LIBOR rates, the exchange rate, and cap volatilities - are obtained from the domestic country (U.S.) and foreign country (U.K.) between June 30, 2006, and July 1, 2008. The current time $\tau$ is set as July 12008 .

The term structures of the forward LIBOR rates of the two countries are plotted in Figure 1 in Appendix C. In Figure 2, the volatilities structures implied in cap markets are drawn comparatively.

In Table 1, the short-maturity notes are considered. The maturity of QFRNs is 180 days, and each compound 
period comprises 30 days $\left(N_{i}=30, \forall i\right)$. In other words, there are six coupon payment dates in the life of the notes. The number of days in a year is $360\left(D_{i}=360, \forall i\right)$. The current time $\tau$ is the coupon payment date $T_{0}$ $\left(\tau=T_{0}, N_{1}^{+}=30\right)$. The interest spread $\Delta_{i j}$ is $2 \%$. Three different corridor levels are examined, and the whole set of coupon payments are listed by three types of QFRNs.

In Table 2, the long-maturity notes are evaluated. The two-year QFRNs have four coupon payment dates, and coupons are paid every half-year $\left(N_{i}=180, \forall i\right)$. The number of days in a year is $360\left(D_{i}=360, \forall i\right)$. The current time $\tau$ is the coupon payment date $T_{0}\left(\tau=T_{0}, N_{1}^{+}=180\right)$. The interest spread $\Delta_{i j}$ is also $2 \%$.

The pricing results of Proposition 3 given in Tables 1 and 2 are listed with the results of the Monte Carlo simulation and standard errors. The results show that the derived formulae of QFRNs are sufficiently accurate compared to the Monte Carlo simulation of both in short- and long-maturity notes. At different levels of corridors, the pricing model performs robustly in the empirical study. The overall results reveal that the pricing model of QFRNs is a good substitute for simulation and exhibits the characteristic of time-efficiency, thereby avoiding the problem of a time-consuming simulation.

Table 1. 180-day coupon payments of QFRNs

\begin{tabular}{|c|c|c|c|c|c|c|c|c|}
\hline \multicolumn{2}{|c|}{ Corridor(\%) } & \multirow{2}{*}{$\frac{C 1}{0.00518}$} & \multirow{2}{*}{$\frac{C 2}{0.00116}$} & \multirow{2}{*}{$\frac{C 3}{0.00213}$} & \multirow{2}{*}{$\frac{C 4}{0.00242}$} & \multirow{2}{*}{$\frac{C 5}{0.00145}$} & \multirow{2}{*}{$\frac{C 6}{0.00150}$} & \multirow{2}{*}{$\frac{\text { QFRN }}{0.99937}$} \\
\hline Type I & $\mathrm{AF}$ & & & & & & & \\
\hline \multirow[t]{2}{*}{$5.5-6.0$} & $\mathrm{MC}$ & 0.00518 & 0.00116 & 0.00212 & 0.00242 & 0.00145 & 0.00150 & 0.99936 \\
\hline & SE & $3.06 \mathrm{E}-06$ & $3.55 \mathrm{E}-06$ & $4.56 \mathrm{E}-06$ & 4.50E-06 & $3.95 \mathrm{E}-06$ & $4.20 \mathrm{E}-06$ & \\
\hline \multirow[t]{3}{*}{$5.0-6.5$} & $\mathrm{AF}$ & 0.00634 & 0.00518 & 0.00554 & 0.00547 & 0.00392 & 0.00405 & 1.01603 \\
\hline & MC & 0.00634 & 0.00518 & 0.00554 & 0.00547 & 0.00392 & 0.00405 & 1.01603 \\
\hline & SE & 2.34E-07 & 3.71E-06 & 3.99E-06 & $3.17 \mathrm{E}-06$ & 4.70E-06 & $5.05 \mathrm{E}-06$ & \\
\hline \multirow[t]{3}{*}{$4.5-7.0$} & $\mathrm{AF}$ & 0.00635 & 0.00655 & 0.00683 & 0.00624 & 0.00549 & 0.00565 & 1.02264 \\
\hline & MC & 0.00635 & 0.00655 & 0.00683 & 0.00624 & 0.00550 & 0.00566 & 1.02265 \\
\hline & SE & $1.26 \mathrm{E}-17$ & $1.24 \mathrm{E}-06$ & 1.62E-06 & $1.41 \mathrm{E}-06$ & 3.74E-06 & 4.11E-06 & \\
\hline Type II & $\mathrm{AF}$ & 0.00488 & 0.00265 & 0.00252 & 0.00214 & 0.00160 & 0.00156 & 1.00088 \\
\hline \multirow[t]{2}{*}{$2.5-3.0$} & MC & 0.00489 & 0.00265 & 0.00252 & 0.00214 & 0.00160 & 0.00156 & 1.00089 \\
\hline & SE & $3.72 \mathrm{E}-06$ & 4.67E-06 & 4.88E-06 & 4.35E-06 & 4.11E-06 & 4.24E-06 & \\
\hline \multirow[t]{3}{*}{$2.0-3.5$} & $\mathrm{AF}$ & 0.00632 & 0.00569 & 0.00576 & 0.00515 & 0.00410 & 0.00409 & 1.01663 \\
\hline & MC & 0.00632 & 0.00569 & 0.00577 & 0.00515 & 0.00409 & 0.00409 & 1.01663 \\
\hline & SE & $5.82 \mathrm{E}-07$ & $3.38 \mathrm{E}-06$ & $3.92 \mathrm{E}-06$ & $3.60 \mathrm{E}-06$ & 4.72E-06 & $5.13 \mathrm{E}-06$ & \\
\hline \multirow[t]{3}{*}{$1.5-4.0$} & $\mathrm{AF}$ & 0.00635 & 0.00651 & 0.00674 & 0.00610 & 0.00545 & 0.00553 & 1.02218 \\
\hline & MC & 0.00635 & 0.00651 & 0.00674 & 0.00610 & 0.00545 & 0.00553 & 1.02218 \\
\hline & SE & $7.78 \mathrm{E}-08$ & $1.48 \mathrm{E}-06$ & 2.03E-06 & $1.98 \mathrm{E}-06$ & $3.79 \mathrm{E}-06$ & 4.33E-06 & \\
\hline Type III & $\mathrm{AF}$ & 0.00311 & 0.00070 & 0.00129 & 0.00147 & 0.00089 & 0.00094 & 0.99392 \\
\hline \multirow[t]{2}{*}{$5.5-6.0$} & MC & 0.00311 & 0.00070 & 0.00128 & 0.00147 & 0.00089 & 0.00094 & 0.99391 \\
\hline & SE & $1.65 \mathrm{E}-06$ & $1.94 \mathrm{E}-06$ & $2.50 \mathrm{E}-06$ & $2.49 \mathrm{E}-06$ & $2.25 \mathrm{E}-06$ & $2.43 \mathrm{E}-06$ & \\
\hline \multirow[t]{3}{*}{$5.0-6.5$} & $\mathrm{AF}$ & 0.00381 & 0.00314 & 0.00337 & 0.00331 & 0.00241 & 0.00253 & 1.00409 \\
\hline & MC & 0.00381 & 0.00314 & 0.00337 & 0.00331 & 0.00241 & 0.00253 & 1.00409 \\
\hline & SE & $1.22 \mathrm{E}-07$ & $2.08 \mathrm{E}-06$ & $2.28 \mathrm{E}-06$ & $1.88 \mathrm{E}-06$ & $2.76 \mathrm{E}-06$ & 2.97E-06 & \\
\hline \multirow[t]{3}{*}{$4.5-7.0$} & $\mathrm{AF}$ & 0.00381 & 0.00398 & 0.00415 & 0.00378 & 0.00338 & 0.00353 & 1.00814 \\
\hline & MC & 0.00381 & 0.00398 & 0.00415 & 0.00378 & 0.00338 & 0.00353 & 1.00814 \\
\hline & SE & 7.20E-18 & $7.95 \mathrm{E}-07$ & 1.12E-06 & $1.05 \mathrm{E}-06$ & 2.19E-06 & $2.54 \mathrm{E}-06$ & \\
\hline
\end{tabular}

Note. a. QFRNs are 180-days and each compound period is 30 days $\left(N_{i}=30, \forall i\right)$. The current time $\tau$ is the coupon payment date $T_{0}$ $\left(\tau=T_{0}, N_{1}^{+}=30\right)$. The number of days in a year is $360\left(D_{i}=360, \forall i\right)$. The interest spread $\Delta_{\Delta_{i j}}$ is $2 \%$.

b. AF stands for the pricing result using Proposition 3. MC and SE represent, respectively, the numerical solution using Monte Carlo simulation with 50,000 random paths and standard errors. $C_{i}$ denotes the $i$ th coupon payment and QFRN is the pricing result of these notes. 
Table 2. 2-year coupon payments of QFRNs

\begin{tabular}{|c|c|c|c|c|c|c|}
\hline Corridor $(\%)$ & & $\mathrm{C} 1$ & $\mathrm{C} 2$ & $\mathrm{C} 3$ & $\mathrm{C} 4$ & QFRN \\
\hline \multirow[t]{3}{*}{ Type I 4.0-6.0 } & $\mathrm{AF}$ & 0.01220 & 0.01906 & 0.01584 & 0.01475 & 0.99606 \\
\hline & MC & 0.01218 & 0.01907 & 0.01585 & 0.01476 & 0.99608 \\
\hline & SE & $1.05 \mathrm{E}-05$ & 1.19E-05 & $1.12 \mathrm{E}-05$ & $1.14 \mathrm{E}-05$ & \\
\hline \multirow[t]{3}{*}{$3.0-7.0$} & $\mathrm{AF}$ & 0.03764 & 0.03128 & 0.02623 & 0.02519 & 1.05456 \\
\hline & MC & 0.03764 & 0.03128 & 0.02622 & 0.02519 & 1.05455 \\
\hline & SE & 5.67E-06 & $9.70 \mathrm{E}-06$ & $1.10 \mathrm{E}-05$ & $1.16 \mathrm{E}-05$ & \\
\hline \multirow[t]{3}{*}{$2.0-8.0$} & $\mathrm{AF}$ & 0.04000 & 0.03667 & 0.03159 & 0.03052 & 1.07300 \\
\hline & MC & 0.04000 & 0.03668 & 0.03160 & 0.03053 & 1.07303 \\
\hline & SE & $1.63 \mathrm{E}-06$ & $6.53 \mathrm{E}-06$ & $9.41 \mathrm{E}-06$ & $1.05 \mathrm{E}-05$ & \\
\hline \multirow[t]{3}{*}{ Type II 3.0-4.0 } & $\mathrm{AF}$ & 0.01731 & 0.01263 & 0.00932 & 0.00833 & 0.98182 \\
\hline & $\mathrm{MC}$ & 0.01733 & 0.01263 & 0.00934 & 0.00833 & 0.98186 \\
\hline & SE & 1.17E-05 & 1.09E-05 & $9.79 \mathrm{E}-06$ & $9.58 \mathrm{E}-06$ & \\
\hline \multirow[t]{3}{*}{$2.0-5.0$} & $\mathrm{AF}$ & 0.03897 & 0.03165 & 0.02432 & 0.02194 & 1.05110 \\
\hline & $\mathrm{MC}$ & 0.03898 & 0.03166 & 0.02433 & 0.02190 & 1.05108 \\
\hline & SE & 4.02E-06 & $9.44 \mathrm{E}-06$ & $1.14 \mathrm{E}-05$ & $1.20 \mathrm{E}-05$ & \\
\hline \multirow[t]{3}{*}{$1.0-6.0$} & $\mathrm{AF}$ & 0.04012 & 0.03730 & 0.03119 & 0.02879 & 1.07162 \\
\hline & $\mathrm{MC}$ & 0.04012 & 0.03731 & 0.03120 & 0.02879 & 1.07164 \\
\hline & SE & $9.89 \mathrm{E}-07$ & 5.97E-06 & $9.64 \mathrm{E}-06$ & $1.09 \mathrm{E}-05$ & \\
\hline \multirow[t]{3}{*}{ Type III 4.0-6.0 } & $\mathrm{AF}$ & 0.00738 & 0.01238 & 0.01148 & 0.01124 & 0.97670 \\
\hline & MC & 0.00738 & 0.01238 & 0.01148 & 0.01125 & 0.97672 \\
\hline & SE & $5.79 \mathrm{E}-06$ & 7.16E-06 & 7.98E-06 & 8.59E-06 & \\
\hline \multirow[t]{3}{*}{$3.0-7.0$} & $\mathrm{AF}$ & 0.02279 & 0.02021 & 0.01896 & 0.01919 & 1.01537 \\
\hline & $\mathrm{MC}$ & 0.02279 & 0.02021 & 0.01896 & 0.01921 & 1.01538 \\
\hline & SE & 3.09E-06 & $6.10 \mathrm{E}-06$ & $8.06 \mathrm{E}-06$ & $9.00 \mathrm{E}-06$ & \\
\hline \multirow[t]{3}{*}{$2.0-8.0$} & $\mathrm{AF}$ & 0.02422 & 0.02361 & 0.02275 & 0.02321 & 1.02801 \\
\hline & MC & 0.02422 & 0.02361 & 0.02275 & 0.02320 & 1.02800 \\
\hline & SE & $8.97 \mathrm{E}-07$ & 4.63E-06 & 7.26E-06 & 8.39E-06 & \\
\hline
\end{tabular}

Note. a. QFRNs are 2 years and coupons are paid every half-year $\left(N_{i}=180, \forall i\right)$. The current time $\mathrm{T}$ is the coupon payment date $T_{0}$ $\left(\tau=T_{0}, N_{1}^{+}=180\right)$. The number of days in a year is $360\left(D_{i}=360, \forall i\right)$. The interest spread $\Delta_{i j}$ is $2 \%$.

b. AF stands for pricing result using Proposition 3. MC and SE represent the numerical solution using Monte Carlo simulation with 50,000 random paths and standard errors, respectively. $C_{i}$ denotes the $i$ th coupon payment and QFRN is the pricing result of these notes.

\section{Conclusion}

We have derived the analytical valuation formulae for three types of QFRNs. The pricing model is a multifactor framework based on domestic and foreign LIBOR rates and the foreign exchange rate. The valuation formulae are easy to implement practically and the model parameters can be acquired easily from market quantities. The derived formulae are analytically tractable, sufficiently accurate and robust, and suitable for pricing QFRNs in practice with the closed-form pricing theorem.

\section{References}

Black, F. (1976). The Pricing of Commodity Contracts. Journal of Financial Economics, 3, 167-179. http://dx.doi.org/10.1016/0304-405X(76)90024-6

Brace, A., Gatarek, D., \& Musiela, M. (1997). The Market Model of Interest Rate Dynamics. Mathematical Finance, 7, 127-155. http://dx.doi.org/10.1111/1467-9965.00028

Eberlein, E., \& Kluge, W. (2006). Valuation of floating range notes in Lévy term structure model. Mathematical Finance, 16, 237-254. http://dx.doi.org/10.1111/j.1467-9965.2006.00270.x

Heath, D., Jarrow, R., \& Morton, A. (1992). Bond Pricing and the Term Structure of Interest Rates: A New

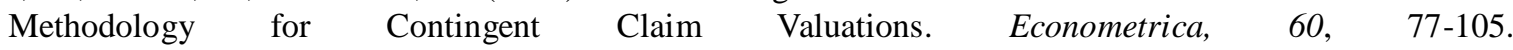
http://dx.doi.org/10.2307/2951677

Liao, S. L., \& Hsu, P. P. (2009). Pricing and Hedging of Quanto Range Accrual Notes under Guassian HJM with Cross-Currency Lévy Processes. Journal of Futures Markets, 29, 973-998. 
http://dx.doi.org/10.1002/fut.20396

Miltersen, K. R., Sandmann, K., \& Sondermann, D. (1997). Closed form solutions for term structure derivatives with log-normal interest rates. The Journal of Finance, 52, 409-430. http://dx.doi.org/10.2307/2329571

Navatte, P., \& Quittard-Pinon, F. (1999). The valuation of interest rate digital options and range notes revisited. European Financial Management, 5, 425-440. http://dx.doi.org/10.1111/1468-036X.00103

Nunes, J. P. V. (2004). Multifactor valuation of floating range notes. Mathematical Finance, $14,79-97$. http://dx.doi.org/10.1111/j.0960-1627.2004.00182.x

Rebonato, R. (1999). On the Simultaneous Calibration of Multifactor Lognormal Interest Rate Models to Black Volatilities and to the Correlation Matrix. Journal of Computational Finance, 2, 5-27.

Turnbull, S. (1995). Interest rate digital options and range notes. Journal of Derivatives, 3, 92-101. http://dx.doi.org/10.3905/jod.1995.407931

Wu, T. P., \& Chen, S. N. (2007). Cross-currency Equity Swap in the BGM Model. Journal of Derivatives, 15, 60-76. http://dx.doi.org/10.3905/jod.2007.699046

Wu, T. P., \& Chen, S. N. (2008). Valuation of Floating Range Notes in a LIBOR Market Model. Journal of Futures Markets, 28, 697-710. http://dx.doi.org/10.1002/fut.20310

\section{Note}

Note 1 . The derivation is available upon request from the authors.

\section{Appendix A}

\section{Proof of Proposition 1}

According to the definition of a DDRO in equation (10), the final payoff is given by

$$
\operatorname{DDRO}\left(T^{+}, T^{+}, H, k\right)=\left\{\begin{array}{cc}
1, & H^{L} \leq L_{k}(T, T, \delta) \leq H^{U} \\
0, & \text { otherwise }
\end{array}\right.
$$

Under the forward martingale measure $Q^{T^{+}}$, the no-arbitrage value of a DDRO at current time $\tau$ is equal to

$$
\begin{aligned}
\operatorname{DDRO}\left(\tau, T^{+}, H, k\right) & =P_{d}\left(\tau, T^{+}\right) E^{T^{+}}\left[I_{\left\{H^{L} \leq L_{k}(T, T, \delta) \leq H^{U}\right\}} \mid F_{\tau}\right] \\
& =P_{d}\left(\tau, T^{+}\right) Q^{T^{+}}\left[H^{L} \leq L_{k}(T, T, \delta) \leq H^{U} \mid F_{\tau}\right]
\end{aligned}
$$

Next, the dynamics of the forward LIBOR rates $L_{k}(T, T, \delta)$ under the CLMM in Assumption 2 can be expressed in the integral form:

$$
L_{k}(T, T, \delta)=L_{k}(\tau, T, \delta) \exp \left\{\rho_{k}(\tau, T, \delta)-\frac{1}{2} v_{k}(\tau, T)+Z_{k}(\tau, T)\right\}
$$

where $Z_{k}(\tau, T)=\int_{\tau}^{T} \gamma_{k}(t, T) \cdot d W^{T^{+}}(t)$ is a normal variate with variance $v_{k}(\tau, T)$.

The elements $\rho_{k}(\tau, T, \delta)$ and $v_{k}(\tau, T)$ are defined in equations (12)-(15). By substituting (A.2) into (A.1), the time $\tau$ value of a DDRO is derived as follows: 


$$
\begin{aligned}
& \operatorname{DDRO}\left(\tau, T^{+}, H, k\right) \\
& =P_{d}\left(\tau, T^{+}\right) Q^{T^{+}}\left[H^{L} \leq L_{k}(T, T, \delta) \leq H^{U} \mid F_{\tau}\right] \\
& =P_{d}\left(\tau, T^{+}\right) Q^{T^{+}}\left[-\frac{Z_{k}(\tau, T)}{\sqrt{v_{k}(\tau, T)}} \leq \frac{\ln \left(L_{k}(T, T, \delta) / H^{L}\right)+\rho_{k}(\tau, T, \delta)-\frac{1}{2} v_{k}(\tau, T)}{\sqrt{v_{k}(\tau, T)}} \mid F_{\tau}\right] \\
& -P_{d}\left(\tau, T^{+}\right) Q^{T^{+}}\left[-\frac{Z_{k}(\tau, T)}{\sqrt{v_{k}(\tau, T)}} \leq \frac{\ln \left(L_{k}(T, T, \delta) / H^{U}\right)+\rho_{k}(\tau, T, \delta)-\frac{1}{2} v_{k}(\tau, T)}{\sqrt{v_{k}(\tau, T)}} \mid F_{\tau}\right] \\
& =P_{d}\left(\tau, T^{+}\right)\left\{N\left[x\left(H^{L}, k\right)\right]-N\left[x\left(H^{U}, k\right)\right]\right\}
\end{aligned}
$$

\section{Appendix B}

\section{Proof of Proposition 2}

According to the definition of a DARO in equation (16), the final payoff is given by

$$
\operatorname{DARO}\left(T^{+}, T^{+}, H, s, k\right)=\left\{\begin{array}{cc}
L_{s}\left(T^{-}, T^{-}, \eta\right), & H^{L} \leq L_{k}(T, T, \delta) \leq H^{U} \\
0, & \text { otherwise }
\end{array}\right.
$$

Under the forward martingale measure $Q^{T^{+}}$, the no-arbitrage value of a DARO at current time $\tau$ is equal to

$$
\operatorname{DARO}\left(\tau, T^{+}, H, s, k\right)=P_{d}\left(\tau, T^{+}\right) E^{T^{+}}\left[L_{s}\left(T^{-}, T^{-}, \eta\right) I_{\left\{H^{L} \leq L_{k}(T, T, \delta) \leq H^{U}\right\}} \mid F_{\tau}\right]
$$

First, the dynamics of the forward LIBOR rate $L_{s}\left(T^{-}, T^{-}, \eta\right)$ can be expressed analogously to (A.2) and given by

$$
L_{s}\left(T^{-}, T^{-}, \eta\right)=L_{s}\left(\tau, T^{-}, \eta\right) \exp \left\{\rho_{k}\left(\tau, T^{-}, \eta\right)-\frac{1}{2} v_{k}\left(\tau, T^{-}\right)+Z_{k}\left(\tau, T^{-}\right)\right\}
$$

Second, the pricing process of (B.1) involves a change of measure. According to Girsanov's Theorem, a new probability measure $R^{T^{+}}$is defined by the Radon-Nikodym derivative $d R^{T^{+}} / d Q^{T^{+}}$with respect to $Q^{T^{+}}$on the same probability space $(\Omega, \mathbf{F})$. By substituting (B.2) into (B.1) with a change of measure to $R^{T^{+}}$, the equation (B.1) becomes

$$
\begin{aligned}
& \operatorname{DARO}\left(\tau, T^{+}, H, s, k\right) \\
& =P_{d}\left(\tau, T^{+}\right) E^{T^{+}}\left[L_{s}\left(T^{-}, T^{-}, \eta\right) I_{\left\{H^{L} \leq L_{k}(T, T, \delta) \leq H^{U}\right\}} \mid F_{\tau}\right] \\
& =P_{d}\left(\tau, T^{+}\right) L_{s}\left(T^{-}, T^{-}, \eta\right) e^{\rho_{k}\left(\tau, T^{-}, \eta\right)} E^{T^{+}}\left[d R^{T^{+}} / d Q^{T^{+}} I_{\left\{H^{L} \leq L_{k}(T, T, \delta) \leq H^{U}\right\}} \mid F_{\tau}\right] \\
& =P_{d}\left(\tau, T^{+}\right) L_{s}\left(T^{-}, T^{-}, \eta\right) e^{\rho_{k}\left(\tau, T^{-}, \eta\right)} E^{R^{+}}\left[I_{\left\{H^{L} \leq L_{k}(T, T, \delta) \leq H^{U}\right\}} \mid F_{\tau}\right]
\end{aligned}
$$

Similarly, the dynamics of the foreign LIBOR rate $L_{k}(T, T, \delta)$ under the martingale measure $R^{T^{+}}$is given by

$$
L_{k}(T, T, \delta)=L_{k}(\tau, T, \delta) \exp \left\{\varphi_{k}(\tau, T, \delta)-\frac{1}{2} v_{k}(\tau, T)+Z_{k}(\tau, T)\right\}
$$

where $\varphi_{k}(\tau, T, \delta)$ is defined in equations (18) and (19). Again, by substituting (B.4) into (B.3), the time $\tau$ value of a DARO is derived as follows. 


$$
\begin{aligned}
& \operatorname{DARO}\left(\tau, T^{+}, H, s, k\right) \\
& =P_{d}\left(\tau, T^{+}\right) L_{s}\left(T^{-}, T^{-}, \eta\right) e^{\rho_{k}\left(\tau, T^{-}, \eta\right)} E^{R^{+}}\left[I_{\left\{H^{L} \leq L_{k}(T, T, \delta) \leq H^{U}\right\}} \mid F_{\tau}\right] \\
& =P_{d}\left(\tau, T^{+}\right) L_{s}\left(T^{-}, T^{-}, \eta\right) e^{\rho_{k}\left(\tau, T^{-}, \eta\right)} R^{T^{+}}\left[H^{L} \leq L_{k}(T, T, \delta) \leq H^{U} \mid F_{\tau}\right] \\
& =P_{d}\left(\tau, T^{+}\right) L_{s}\left(\tau, T^{-}, \eta\right) e^{\rho_{s}\left(\tau, T^{-}, \eta\right)}\left\{N\left[y\left(H^{L}, k\right)\right]-N\left[y\left(H^{U}, k\right)\right]\right\}
\end{aligned}
$$

\section{Appendix C}

\section{Market Data}

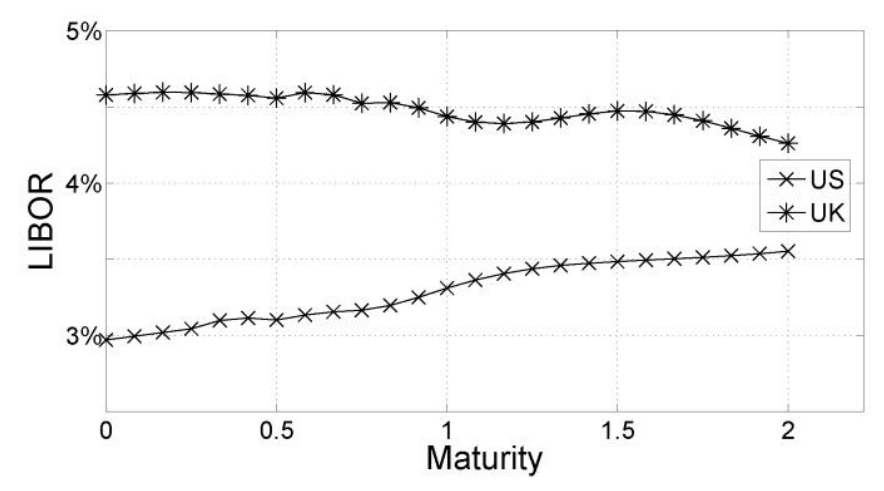

Figure 1. The term structure of interest rate in U.S. and U.K. markets

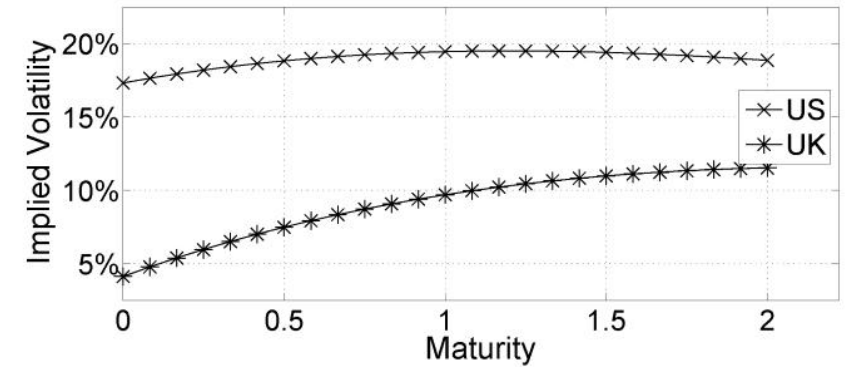

Figure 2. The term structure of volatilities implied in cap markets

\section{Copyrights}

Copyright for this article is retained by the author(s), with first publication rights granted to the journal.

This is an open-access article distributed under the terms and conditions of the Creative Commons Attribution license (http://creativecommons.org/licenses/by/3.0/). 